\title{
Maternal Swimming Exercise During Pregnancy Improves Memory Through Enhancing Neurogenesis and Suppressing Apoptosis via Wnt/ $\beta$-Catenin Pathway in Autistic Mice
}

\author{
Sang-Seo Park ${ }^{1,2}$, Chang-Ju Kim ${ }^{1}$, Seong-Hyun Kim³ ${ }^{3}$,Tae-Woon Kim ${ }^{4}$, Sam-Jun Lee ${ }^{5}$ \\ ${ }^{1}$ Department of Physiology, College of Medicine, Kyung Hee University, Seoul, Korea \\ ${ }^{2}$ School of Health and Kinesiology, University of Nebraska at Omaha, Omaha, NE, USA \\ ${ }^{3}$ College of Culture and Sports, Division of Global Sport Studies, Korea University, Sejong, Korea \\ ${ }^{4}$ Department of Human Health Care, Gyeongsang National University, Jinju, Korea \\ ${ }^{5}$ Department of Sport Rehabilitation, College of Health, Welfare and Education, Tongmyong University, Busan, Korea
}

Purpose: Wnt pathway is closely related to neurodevelopmental process associated with cognitive function. After administration of valproic acid to the pregnant mice, the effect of swimming exercise of pregnant mice on the memory, neuronal production, and apoptosis of pups was studied in relation with $W n t / \beta$-catenin signaling pathway.

Methods: On day 12 of pregnancy, mice were injected subcutaneously with $400-\mathrm{mg} / \mathrm{kg}$ valproic acid. The pregnant mice in the control with swimming exercise group and in the valproic acid injection with swimming exercise group were allowed for swimming for 30 minutes one time per a day, repeated 5 days per a week, during 3 weeks. Step-through avoidance task and Morris water maze task for memory function, immunohistochemistry for 5-bromo-2'-deoxyuridine (BrdU)-positive cells and western blot for brain-derived neurotrophic factor (BDNF), Wnt, $\beta$-catenin, Bcl-2 related X protein (Bax), B-cell lymphoma 2 (Bcl-2), cleaved caspase-3 were carried out.

Results: Maternal swimming exercise during pregnancy improved memory function, increased BDNF expression, and neuronal proliferation in the valproic acid injected pups. Maternal swimming exercise during pregnancy suppressed Wnt expression and phosphorylation of $\beta$-catenin in the valproic acid injected pups. Maternal swimming exercise inhibited Bax and cleaved caspase- 3 expression and increased Bcl-2 expression in the valproic acid injected pups.

Conclusions: Maternal swimming exercise during pregnancy improved memory function by increasing cell proliferation and inhibiting apoptosis through Wnt/ $\beta$-catenin signaling cascade activation in the valproic acid injected pups. Maternal swimming exercise during pregnancy may have a protective effect on factors that induce autism in the fetus.

Keywords: Autism; Swimming; Memory; Wnt/ $\beta$-catenin pathway; Apoptosis

- Fund/Grant Support: This work was supported by the Ministry of Education of the Republic of Korea and the National Research Foundation of Korea (NRF-2018S1A5A2A01038919).

- Research Ethics: All experimental process was approved by the Institutional Animal Care and Use Committee of the Kyung Hee University, and the following approval number, KHUASP(SE)-19-133, was obtained.

- Conflict of Interest: No potential conflict of interest relevant to this article was reported.

\footnotetext{
- HIGHLIGHTS

- Injection of valproic acid to the pregnant maternal rats impaired memory function in the pups.

- Maternal swimming during pregnancy improved memory function via Wnt/ß-catenin activation in the valproic acid injected pups.

- Maternal swimming during pregnancy also enhanced neurogenesis and inhibited apoptosis in the valproic acid injected pups.
}

Corresponding author: Sam-Jun Lee (D) https://orcid.org/0000-0002-8491-3602 Department of Sport Rehabilitation, College of Health, Welfare and Education, Tongmyong University, 428 Sinseon-ro, Nam-gu, Busan 48520, Korea Email: anada23@tu.ac.kr

Submitted: September 5, 2021 / Accepted after revision: October 21, 2021
This is an Open Access article distributed under the terms of the Creative Commons Attribution Non-Commercial License (http://creativecommons.org/licenses/by-nc/4.0/) which permits unrestricted non-commercial use, distribution, and reproduction in any medium, provided the original work is properly cited. 


\section{INTRODUCTION}

Low birth weight and premature birth are implicated in a higher risk of impaired social interaction and communication in adulthood [1]. Motor deficits are a specific subgroup of early autism spectrum disorders [2]. Autism spectrum disorders are neurodevelopmental disorders characterized by repetitive and restrictive behavior in social interaction and communication [3].

The Wnt pathway is closely related to neurodevelopmental process associated and Wnt plays a variety of roles in cells by regulating $\beta$-catenin expression in the brain [4]. The Wnt signaling pathway contributed to neurogenesis and synaptogenesis by regulating downstream of the glycogen synthase kinase 3 beta (GSK-3 $\beta$ )/phosphatidylinositol 3-kinase (PI3K)/protein kinase B (Akt)/cyclic adenosine monophosphate response element-binding protein $[5,6]$. In autism spectrum disorders, signaling pathway of the $\mathrm{Wnt} / \beta$-catenin was up-regulated, and this up-regulated pathway of the Wnt/ $\beta$-catenin induced aerobic glycolysis through activation of 3-phosphoinositide-dependent protein kinase-1 and monocarboxylate lactate transporter 1 [3]. Autism-induced hyperactivation of the Wnt/ $\beta$-catenin signaling cascade led to the activation of hypoxia-induced factor 1- $\alpha$ via the downstream PI3K/Akt pathway [7,8]. Mitochondrial dysfunction induced bipolar disorder and improving mitochondrial function was essential factor for long-term treatment for this disorder [9]. Therefore, the various neurodevelopmental disorders observed in autism may be closely related to overactivation of Wnt/ $\beta$-catenin signaling cascade [3]. In the study of genetic variation, children with autism showed disrupted Wnt $/ \beta$-catenin signaling cascade [10]. When valproic acid, a GSK-3 $\beta$ inhibitor that interferes with the Wnt signaling pathway, was administered to maternal rats, autism appeared in the offspring [11].

Exercise is a major extrinsic factor that positively affects the structural development and functional improvement of the brain throughout life [12]. Exercise regulated neuronal proliferation, differentiation, and maturation by affecting brain-derived neurotrophic factor (BDNF) expression and modulating downstream cellular signal cascades [13]. Exercise protected against neuronal damage through therapeutic and neuroprotective effect for neurodegenerative disorders [14,15]. Exercise regulated Wnt signaling pathway implicated in neuroprotection and synaptic plasticity [16]. Swimming exercise in maternal rats during pregnancy affected offspring brain development, especially in the hippocampus [17]. Maternal exercise during pregnancy improved habituation behavior and spatial working memory and increased BDNF level and new cell formation in the hippocampus of offspring [18].

After administration of valproic acid to pregnant mice, the effect of swimming exercise of pregnant mice on the memory, neuronal production, and apoptosis of pups was studied in association with Wnt/ $\beta$-catenin signaling pathway. Step-through avoidance task and Morris water maze task for memory function, immunohistochemistry for 5-bromo-2'-deoxyuridine (BrdU), and western blotting for BDNF, Wnt, $\beta$-catenin, Bcl-2 related X protein (Bax), B-cell lymphoma 2 (Bcl-2), cleaved caspase-3 were carried out.

\section{MATERIALS AND METHODS}

\section{Experiment Animals}

All experimental process was verified by the Institutional Animal Care and Use Committee of the Kyung Hee University, and the following approval number, KHUASP(SE)-19-133, was obtained. Bl6 mice in 10 weeks old (12 males, 24 females) were used for mating. The female mice were intraperitoneally injected with BrdU (100 mg/kg, Sigma Chemical Co., St. Louis, MO, USA) once a day, 5 days, before mating. After birth, the pups were classified into 4 groups ( $\mathrm{n}=10$ in each group) according to the maternal conditions: control group, control with swimming exercise group, valproic acid injection group, and valproic acid injection with swimming exercise group.

\section{Valproic Acid Injection}

To induce autism-like pup model, valproic acid (Sigma-Aldrich Chemical Co., St. Louis, MO, USA) was dissolved in saline at $0.1-\mathrm{mL} / \mathrm{kg}$ concentration, and $400-\mathrm{mg} / \mathrm{kg}$ valproic acid was administered subcutaneously to mice on day 12 of pregnancy, as the previous mentioned method [19].

\section{Swimming Exercise Protocol}

As per the method described earlier [20], the pregnant mice in the control with swimming exercise group and in the valproic acid injection with swimming exercise group were allowed for swimming for 30 minutes one time per a day, repeated 5 days per a week, for 3 continuous weeks.

\section{Step-Through Avoidance Task}

As per the method described earlier [21], step-through avoid- 
ance task was done to detect short-term memory. Mice were put in the light compartment in a box divided into dark compartment and light compartment. The door that divides the two compartments opened and the mouse entered the dark compartment and then the door closed. Immediately after, foot shock of $0.2 \mathrm{~mA}$ current and 2-seccond duration was applied once. After 24 hours, the mouse was again put in the light compartment and the time from reopening the door to reentering the dark compartment was measured (latency). Any time greater than 300 seconds was recorded as 300 seconds.

\section{Morris Water Maze Task}

According to the method described earlier [19], Morris water maze task was performed to measure spatial working memory. After immersing the mouse in a water bath, the mouse was made to find a platform placed under the water. When the mouse could not find the platform within 60 seconds, the experimenter using hand guided the mouse to the platform. Upon arrival at the platform, the mouse was left to stay on the platform for 30 seconds. Training using the platform was performed 3 times a day during 5 consecutive days. Measurement of time in probe quadrant around the platform via video tracking was performed 24 hours after training sessions. Memory retention was judged by the time in probe quadrant around the previous platform.

\section{Tissue Preparation}

The mice were sacrificed after the last Morris water maze task, according to the method described earlier [22]. After anesthetizing by intraperitoneal injection of Zoletil $50(10 \mathrm{mg} / \mathrm{kg}$, Vibac Laboratories, Carros, France), the mice were infused with $50 \mathrm{mM}$ phosphate-buffered saline via heart. The mice were treated with $4 \%$ paraformaldehyde in $100 \mathrm{mM}$ phosphate buffer for fixation. The brains were removed, after that, the brains were treated with same fixation solution. The brains were incubated in the $30 \%$ sucrose solution. Sagittal sections with a thickness of $40 \mu \mathrm{m}$ were prepared by freezing microtome (Leica, Nussloch, Germany).

\section{Immunohistochemistry for BrdU}

As per the method described earlier [23], immunohistochemistry was performed to detect the number of BrdU- positive cells in the dentate gyrus of hippocampus. Average 10 sections containing hippocampal dentate gyrus were selected in each group. The sections were applied to $1 \% \mathrm{H}_{2} \mathrm{O}_{2}$ for 30 minutes, and then treated with BrdU antibody (1:500; Roche, Mannheim, Germany) overnight. The sections were treated with biotinylated antimouse secondary antibody (Vector Laboratories, Burlingame, CA, USA) for 1 hour 30 minutes. To visualize the BrdU, the sections were applied to the $50 \mathrm{mM}$ Tris- $\mathrm{HCl}(\mathrm{pH}, 7.6)$ containing $0.03 \%$ diaminobenzidine, $40-\mathrm{mg} / \mathrm{mL}$ nickel chloride, and $0.03 \% \mathrm{H}_{2} \mathrm{O}_{2}$ for 5 minutes. Mouse antineuronal nuclei antibody (1:1,000; Chemicon International, Temecula, CA, USA) was processed following BrdU staining. The sections were incubated with a biotinylated anti-mouse secondary antibody, and then applied to a reaction mixture containing $0.03 \%$ diaminobenzidine and $0.03 \% \mathrm{H}_{2} \mathrm{O}_{2}$ for 5 minutes. The sections were placed on gelatin-coated slides, and then after air drying overnight, Permount (Thermo Fisher Scientific, Waltham, MA, USA) as used for coverslips mounting onto the slides.

\section{Western Blot Analysis}

Western blotting was done, as per the earlier mentioned method $[24,25]$. Hippocampal tissues were dissolved using lysis buffer of protein. Color-determining protein assay kit (Bio-Rad, Hercules, CA, USA) detected protein concentration. After separation of $20 \mu \mathrm{g}$ of protein using a sodium dodecyl sulfatepolyacrylamide gel, it was moved to a nitrocellulose membrane (Schleicher \& Schuell GmbH, Dassel, Germany). Anti-mouse $\beta$-actin antibody (1:1,000; Santa Cruz Biotechnology, CA, USA), anti-mouse Bcl-2 antibody (1:1,000; Cell Signaling Technology, Inc., Danvers, MS, USA), anti-rabbit Bax antibody (1:1,000; Cell Signaling Technology), anti-rabbit cleaved caspase-3 antibody (1:1,000; Cell Signaling Technology), anti-rabbit Wnt antibody (1:1,000; Cell Signaling Technology), antirabbit $\beta$-catenin antibody (1:1,000; Cell Signaling Technology), and anti-rabbit BDNF antibody (1:1,000; BDNF; 1:1000; Cell Signaling Technology) were chosen as the primary antibodies. Horseradish peroxidase-conjugated anti-mouse antibodies (1:3,000; Santa Cruz Biotechnology) for $\beta$-actin and Bcl-2 and horseradish peroxidase-conjugated anti-rabbit antibodies (1:5,000; Santa Cruz Biotechnology) for Bax, cleaved caspase-3, Wnt, $\beta$-catenin, and BDNF were chosen as the secondary antibodies. The determination of quantity of bands was done by enhanced chemiluminescence detection system (Amersham Pharmacia Biotech GmbH, Freiburg, Germany).

\section{Statistical Analysis}

Data analysis was done using IBM SPSS Statistics ver. 26.0 (IBM Co., Armonk, NY, USA). One-way analysis of variance with 
Tukey post hoc test was done for comparison among the groups. The results were expressed as the mean \pm standard error of the mean. P-value less than 0.05 was considered significant.

\section{RESULTS}

\section{Short-term Memory and Spatial Working Memory}

Fig. 1 shows the results of step-through avoidance task and Morris water maze task. The latency of step-through task and the time in probe quadrant around the platform of Morris water maze task were shortened in the pups of the valproic acid

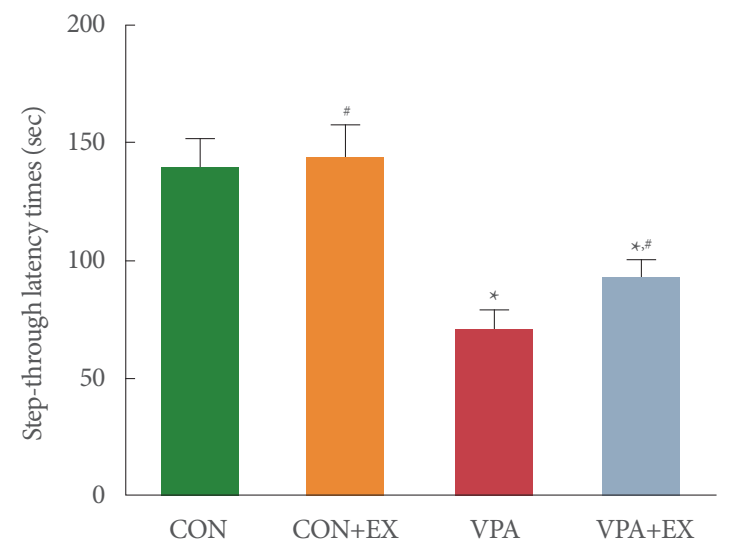

injection in comparison with the control pups $(\mathrm{P}<0.05)$. Swimming exercise during pregnancy lengthened the latency of stepthrough avoidance task and the time in probe quadrant around the platform of Morris water maze task in the pups of the valproic acid injection $(\mathrm{P}<0.05)$.

\section{Number of BrdU-positive Cells}

The number of BrdU-positive cells in the hippocampal dentate gyrus is presented in the Fig. 2. The number of BrdU-positive cells in the hippocampal dentate gyrus was suppressed in the pups of the valproic acid injection in comparison with the con-

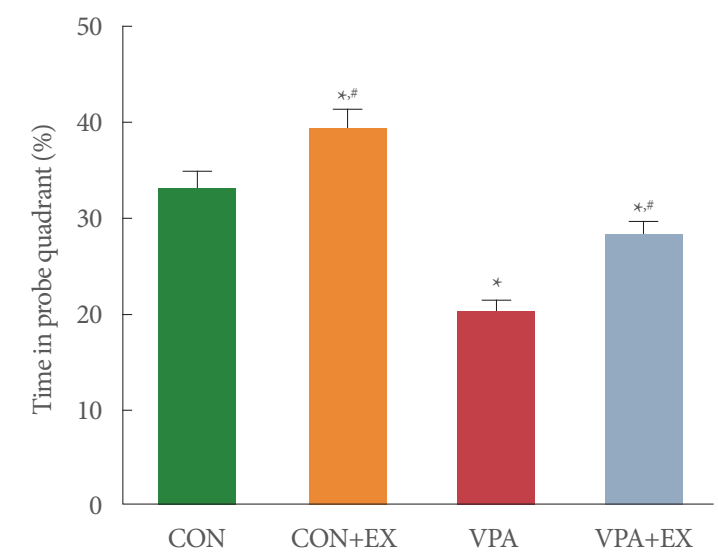

Fig. 1. Influence of swimming exercise on short-term memory and spatial working memory during pregnancy. Left panel: stepthrough avoidance task. Right panel: Morris water maze task. CON, control group; $\mathrm{CON}+\mathrm{EX}$, control with swimming exercise group; $\mathrm{VAP}$, valproic acid injection group; $\mathrm{VAP}+\mathrm{EX}$, valproic acid injection with swimming exercise group. ${ }^{\star} \mathrm{P}<0.05$ in comparison with the control group. ${ }^{*} \mathrm{P}<0.05$ in comparison with valproic acid injection group.
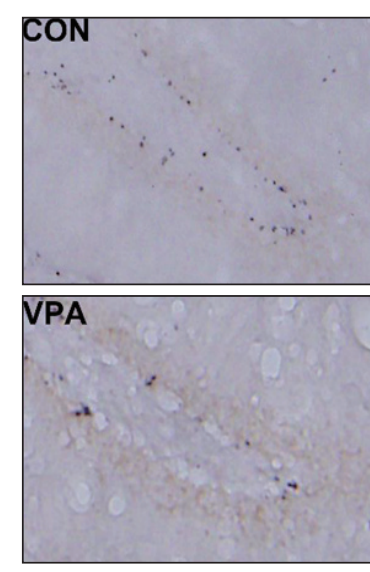

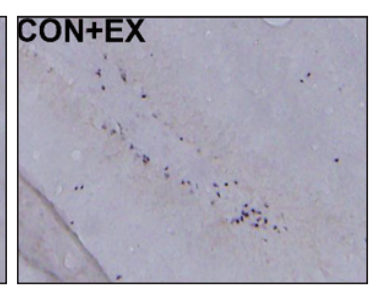

VPA+EX

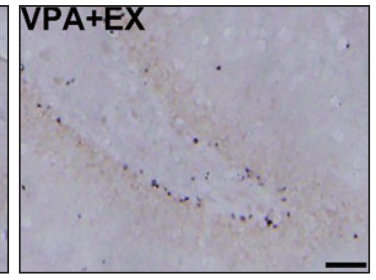

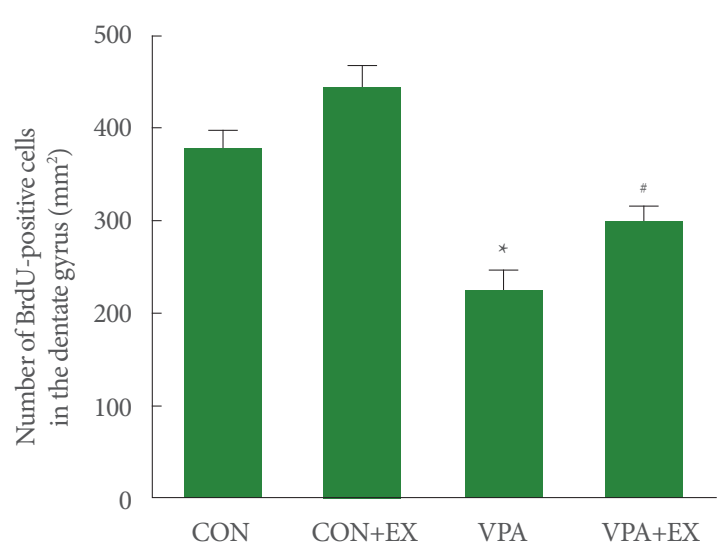

Fig. 2. Influence of swimming exercise during pregnancy on new cell formation in the hippocampal dentate gyrus. Left panel: photomicrographs of 5-bromo-2'-deoxyuridine (BrdU)-positive cells. The scale bar indicates $50 \mu \mathrm{m}$. Right panel: the number of BrdU-positive cells in each group. CON, control group; $\mathrm{CON}+\mathrm{EX}$, control with swimming exercise group; VAP, valproic acid injection group; $\mathrm{VAP}+\mathrm{EX}$, valproic acid injection with swimming exercise group. ${ }^{\star} \mathrm{P}<0.05$ in comparison with the control group. ${ }^{*} \mathrm{P}<0.05$ in comparison with valproic acid injection group. 
trol pups $(\mathrm{P}<0.05)$. Swimming exercise during pregnancy enhanced the number of BrdU-positive cells in the pups of the valproic acid injection $(\mathrm{P}<0.05)$.

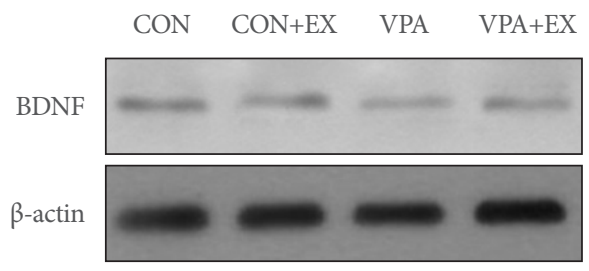

\section{BDNF Expression}

Fig. 3 shows the expression of BDNF in the hippocampus. BDNF expression was suppressed in the pups of the valproic

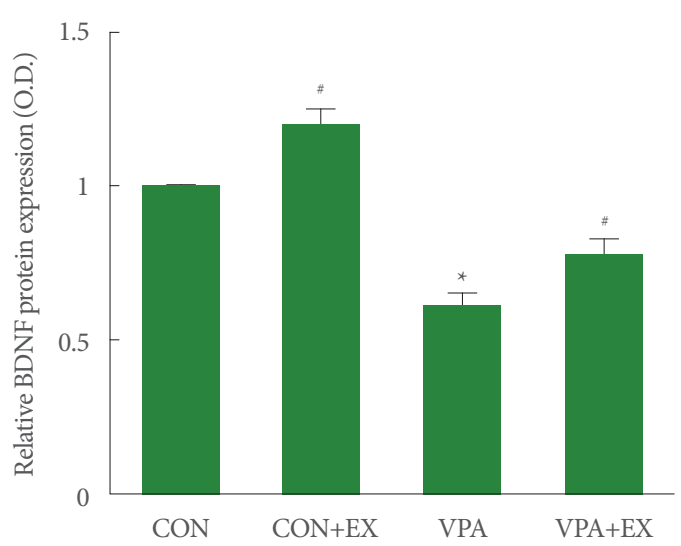

Fig. 3. Influence of swimming exercise during pregnancy on brain-derived neurotrophic factor (BDNF) in the hippocampus. Left panel: representative BDNF expressions. Right panel: relative expression of BDNF in each group. CON, control group; CON+EX, control with swimming exercise group; VAP, valproic acid injection group; VAP+EX, valproic acid injection with swimming exercise group. ${ }^{\star} \mathrm{P}<0.05$ in comparison with the control group. ${ }^{*} \mathrm{P}<0.05$ in comparison with valproic acid injection group
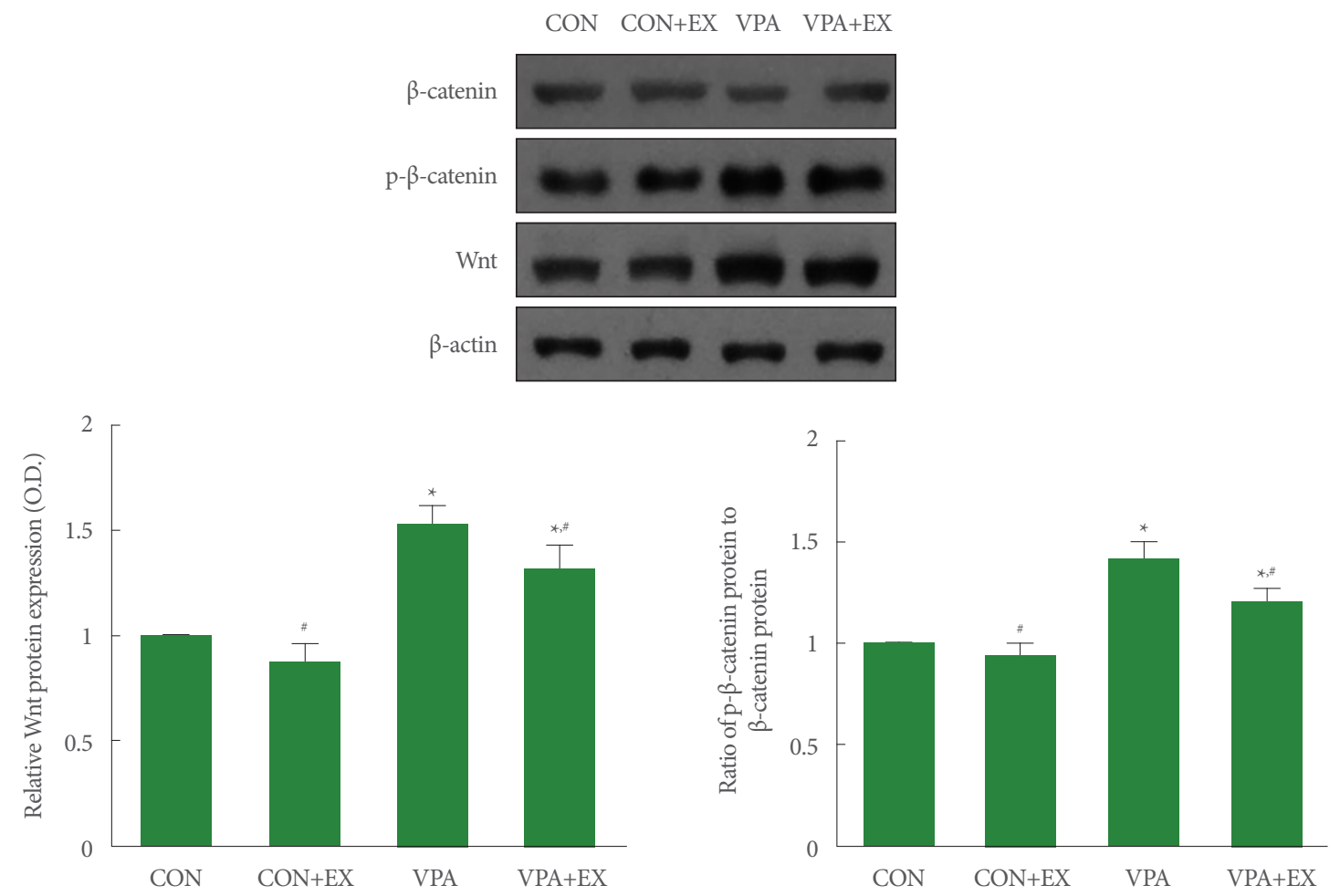

Fig. 4. Influence of swimming exercise on Wnt/ $\beta$-catenin pathway in the hippocampus. Upper panel: representative Wnt and $\beta$-catenin expressions. Lower left panel: relative expression of Wnt in each group. Lower right panel: relative expression of phosphorylated $\beta$-catenin ( $\mathrm{p}-\beta$-catenin) in each group. $\mathrm{CON}$, control group; $\mathrm{CON}+\mathrm{EX}$, control with swimming exercise group; VAP, valproic acid injection group; $\mathrm{VAP}+\mathrm{EX}$, valproic acid injection with swimming exercise group. ${ }^{\star} \mathrm{P}<0.05$ in comparison with the control group. ${ }^{*} \mathrm{P}<0.05$ in comparison with valproic acid injection group. 

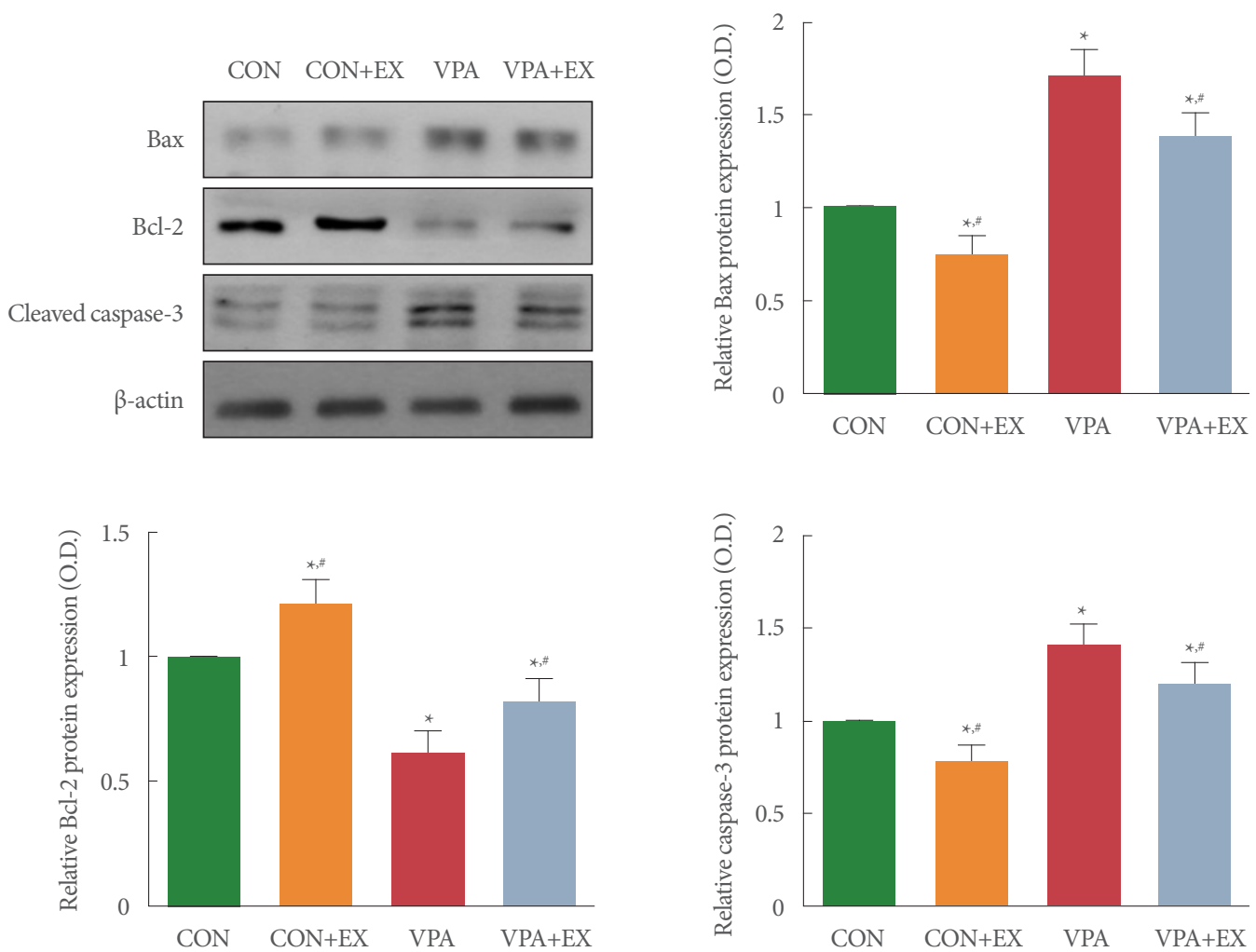

Fig. 5. Influence of swimming exercise on Bcl-2 related X protein (Bax), B-cell lymphoma 2 (Bcl-2), and cleaved caspase-3 expressions in the hippocampus. Upper left panel: representative Bax, Bcl-2, and cleaved caspase-3 expressions. Upper right panel: relative expression of Bax in each group. Lower left panel: relative expression of Bcl-2 in each group. Lower right panel: relative expression of caspase-3 in each group. CON, control group; $\mathrm{CON}+\mathrm{EX}$, control with swimming exercise group; VAP, valproic acid injection group; $\mathrm{VAP}+\mathrm{EX}$, valproic acid injection with swimming exercise group. ${ }^{\star} \mathrm{P}<0.05$ in comparison with the control group. ${ }^{*} \mathrm{P}<0.05$ in comparison with valproic acid injection group.

acid injection in comparison with the control pups $(\mathrm{P}<0.05)$. Swimming exercise during pregnancy enhanced BDNF expression in the pups of the valproic acid injection $(\mathrm{P}<0.05)$.

\section{Wnt/ $\beta$-Catenin Expressions}

Fig. 4 shows the expression of Wnt and phosphorylation of $\beta$-catenin in the hippocampus. Wnt expression and $\beta$-catenin phosphorylation were enhanced in the pups of the valproic acid injection in comparison with the control pups $(\mathrm{P}<0.05)$. Swimming exercise during pregnancy suppressed Wnt expression and $\beta$-catenin phosphorylation in the pups of the valproic acid injection $(\mathrm{P}<0.05)$.

\section{Bax, Bcl-2, Cleaved Caspase-3 Expressions}

Fig. 5 shows the expression of Bax, Bcl-2, and cleaved caspase- 3 in the hippocampus. Bax and cleaved caspase- 3 expression was enhanced and Bcl-2 expression was decreased in the pups of the valproic acid injection in comparison with the control pups $(\mathrm{P}<0.05)$. Swimming exercise during pregnancy suppressed Bax and cleaved caspase- 3 expressions and enhanced Bcl-2 expression in the pups of the valproic acid injection $(\mathrm{P}<0.05)$.

\section{DISCUSSION}

Many studies reported that reduced memory function was observed in the valproic acid-induced autism model $[2,11,19]$. Autism is a neurodevelopmental disorder characterized by repetitive behavior, leading to poor social interaction and cognitive dysfunction [26,27]. Exposure to valproic acid delayed growth and development and caused cognitive impairment or neurological disorders [28]. Kataoka et al. [29] reported that administering of valproic acid during pregnancy resulted in social interaction deficits, anxiety-like behavior, and memory impairment at age 4-8 weeks in mice. In the current study, valpro- 
ic acid administration during pregnancy disturbed memory function in the valproic acid injection pups. Meanwhile, maternal swimming exercise during pregnancy improved memory function in the valproic acid injected pups.

Valproic acid exposure during pregnancy increased apoptotic nerve cell death in the neocortex and decreased neuronal cell generation in the ganglionic eminence [29]. Enhancement of neuronal generation via BDNF signaling cascade activated by treadmill running improved short-term memory [21]. In the current study, valproic acid administration during pregnancy suppressed neuronal proliferation in the valproic acid injection pups. Meanwhile, maternal swimming exercise during pregnancy enhanced neuronal proliferation in the valproic acid injected pups.

Mice exposed to valproic acid revealed lower level of cortical expression of BDNF mRNA and displayed delayed physical development and impaired pre-weaning social behavior [30]. Treadmill running improved BDNF expression, which alleviated anxiety, depression and memory impairment caused by nicotine withdrawal in rats [23]. In the current study, valproic acid administration during pregnancy reduced BDNF level in the valproic acid injected pups. Meanwhile, maternal swimming exercise during pregnancy effectively enhanced BDNF level in the valproic acid injected pups.

In the autism spectrum disorders, the Wnt $/ \beta$-catenin signaling cascade was activated, and this $\mathrm{Wnt} / \beta$-catenin signaling cascade activation induced aerobic glycolysis that was less efficient for adenosine triphosphate production [3]. In the mature nervous system, the Wnt/ $\beta$-catenin signaling cascade involved in neuroprotection and synaptic plasticity was activated by moderate exercise [16]. Treadmill exercise of pups exerted antidepressant effect on the maternal separation rat pups through activation of Wnt signaling pathway [25]. In the current study, valproic acid administration during pregnancy enhanced Wnt expression and $\beta$-catenin phosphorylation in the valproic acid injected pups. Meanwhile, maternal swimming exercise suppressed Wnt expression and $\beta$-catenin phosphorylation in the valproic acid injected pups.

Valproic acid acted directly on neurons and indirectly through glial cells, affecting neuron survival/apoptosis balance and synaptic plasticity [31]. Social isolation in the old rats enhanced Bax expression and inhibited Bcl-2 expression, thereby showing acceleration of apoptosis. Meanwhile swimming exercise in the old rats suppressed Bax expression enhanced Bcl-2 expression, showing inhibition of apoptosis [20]. Maternal exercise sup- pressed Bax and caspase- 3 expression and increased Bcl-2 expression in the pups born from the obese and old maternal rats [32]. In the current study, valproic acid administration during pregnancy increased Bax and cleaved caspase- 3 expression and inhibited Bcl-2 expression in the valproic acid injected pups. Meanwhile, maternal swimming exercise inhibited Bax and cleaved caspase- 3 expression and enhanced Bcl-2 expression in the valproic acid injected pups.

In conclusion, maternal exercise during pregnancy improved memory function by increasing cell proliferation and inhibiting apoptosis through Wnt/ $\beta$-catenin signaling cascade activation in the valproic acid injected pups. Maternal swimming exercise during pregnancy may have a protective effect on factors that induce autism in the fetus.

\section{AUTHOR CONTRIBUTION STATEMENT}

- Conceptualization: SSP

- Data curation: SHK, TWK

- Formal analysis: $S H K, T W K$

- Funding acquisition: $S J L$

- Methodology: SSP

- Project administration: $S J L$

- Visualization: $C J K$

-Writing-original draft: SSP

-Writing-review \& editing: $C J K$

\section{ORCID}

Sang-Seo Park

0000-0001-7033-9630

Chang-Ju Kim

0000-0003-4749-5795

Seong-Hyun Kim

0000-0002-6522-4203

Tae-Woon Kim

0000-0001-8832-0874

Sam-Jun Lee

0000-0002-8491-3602

\section{REFERENCES}

1. Mahoney AD, Minter B, Burch K, Stapel-Wax J. Autism spectrum disorders and prematurity: a review across gestational age subgroups. Adv Neonatal Care 2013;13:247-51.

2. Esposito G, Venuti P, Maestro S, Muratori F. An exploration of symmetry in early autism spectrum disorders: analysis of lying. Brain Dev 2009;31:131-8.

3. Vallée A, Vallée JN. Warburg effect hypothesis in autism spectrum disorders. Mol Brain 2018;11:1. 
4. Clevers H, Nusse R. Wnt/ $\beta$-catenin signaling and disease. Cell 2012;149:1192-205.

5. Huang J, Nguyen-McCarty M, Hexner EO, Danet-Desnoyers G, Klein PS. Maintenance of hematopoietic stem cells through regulation of Wnt and mTOR pathways. Nat Med 2012;18:1778-85.

6. Chen J, Alberts I, Li X. Dysregulation of the IGF-I/PI3K/AKT/ mTOR signaling pathway in autism spectrum disorders. Int J Dev Neurosci 2014;35:35-41.

7. Sun Q, Chen X, Ma J, Peng H, Wang F, Zha X, et al. Mammalian target of rapamycin up-regulation of pyruvate kinase isoenzyme type M2 is critical for aerobic glycolysis and tumor growth. Proc Natl Acad Sci U S A 2011;108:4129-34.

8. Wang LR, Kim SH, Baek SS. Effects of treadmill exercise on the anxiety-like behavior through modulation of GSK3 $\beta / \beta$-catenin signaling in the maternal separation rat pup. J Eexer Rehabil 2019; 15:206-12.

9. Quiroz JA, Gray NA, Kato T, Manji HK. Mitochondrially mediated plasticity in the pathophysiology and treatment of bipolar disorder. Neuropsychopharmacology 2008;33:2551-65.

10. Krumm N, O’Roak BJ, Shendure J, Eichler EE. A de novo convergence of autism genetics and molecular neuroscience. Trends Neurosci 2014;37:95-105.

11. Go HS, Kim KC, Choi CS, Jeon SJ, Kwon KJ, Han SH, et al. Prenatal exposure to valproic acid increases the neural progenitor cell pool and induces macrocephaly in rat brain via a mechanism involving the GSK-3 $\beta / \beta$-catenin pathway. Neuropharmacology 2012;63:1028-41.

12. Voss MW, Vivar C, Kramer AF, van Praag H. Bridging animal and human models of exercise-induced brain plasticity. Trends Cogn Sci 2013;17:525-44.

13. Vivar C, Potter MC, van Praag H. All about running: synaptic plasticity, growth factors and adult hippocampal neurogenesis. Curr Top Behav Neurosci 2013;15:189-210.

14. Cotman CW, Berchtold NC, Christie LA. Exercise builds brain health: key roles of growth factor cascades and inflammation. Trends Neurosci 2007;30:464-72.

15. Duzel E, van Praag H, Sendther M. Can physical exercise in old age improve memory and hippocampal function? Brain 2016;139(Pt 3):662-73.

16. Bayod S, Menella I, Sanchez-Roige S, Lalanza JF, Escorihuela RM, Camins A, et al. Wnt pathway regulation by long-term moderate exercise in rat hippocampus. Brin Res 2014;1543:38-48.

17. Marcelino TB, de Lemos Rodrigues PI, Miguel PM, Netto CA, Pereira Silva LO, Matté C. Effect of maternal exercise on biochemical parameters in rats submitted to neonatal hypoxia-ischemia.
Brain Res 2015;1622:91-101.

18. Gomes da Silva S, Aparecido de Almeida ASérgio, Fernandes J, Lopim GM, Cabral FR, Scermi DA, et al. Maternal exercise during pregnancy increases BDNF levels and cell numbers in the hippocampal formation but not in the cerebral cortex of adult rat offspring. PLoS One 2016;11:e0147200.

19. Wagner GC, Reuhl KR, Cheh M, McRae P, Halladay AK. A new neurobehavioral model of autism in mice: pre-and postnatal exposure to sodium valproate. J Autism Dev Disorder 2006;36:779-93.

20. Park SS, Park HS, Kim TW, Lee SJ. Effects of swimming exercise on social isolation-induced memory impairment and apoptosis in old rats. J Exerc Rehabil 2020;16:234-41.

21. Kim M, Kim TW, Kim CJ, Shin MS, Hong M, Park HS, et al. Berberine ameliorates brain inflammation in poloxamer 407-induced hyperlipidemic rats. Int Neurourol J 2019;23(Suppl 2):S102-10.

22. JI ES, Kim YM, Ko YJ, Baek SS. Treadmill exercise in obese maternal rats during pregnancy improves short-term memory through neurogenesis in the hippocampus of rat pups. J Exerc Rehabil 2020;16:392-7.

23. Park SS, Shin MS, Park HS, Kim TW, Kim CJ, Lim BV. Treadmill exercise ameliorates nicotine withdrawal-induced symptoms. J Exerc Rehabil 2019;15:383-91.

24. Ko YJ, Kim BK, Ji ES. Treadmill exercise in obese maternal rats during pregnancy improves spatial memory through activation of phosphatidylinositol 3-kinase pathway in the hippocampus of rat pups. J Exerc Rehabil 2020;16:483-8.

25. Lee JM, Kim TW, Park SS, Kim CJ, Shin MS, Kim SH, et al. Wnt signaling pathway is implicated in the alleviating effect of treadmill exercise on maternal separation-induced depression. J Exerc Rehabil 2019;15:200-5.

26. Bölte S, Mahdi S, de Vries P, Granlund M, Robison JE, Shulman C, et al. The Gestalt of functioning in autism spectrum disorder: results of the international conference to develop final consensus International Classification of Functioning, Disability and Health core sets. Autism 2019;23:449-67.

27. Gobbi G, Janri L. Sodium-and magnesium-valproate in vivo modulate glutamatergic and GABAergic synapses in the medial prefrontal cortex. Psychopharmacology (Berl) 2006;185:255-62.

28. Markram K, Markram H. The intense world theory - a unifying theory of the neurobiology of autism. Front Hum Neurosci 2010;4: 224.

29. Kataoka S, Takuma K, Hara Y, Maeda Y, Ago Y, Matsuda T. Autism-like behaviours with transient histone hyperacetylation in mice treated prenatally with valproic acid. Int J Neuropsychopharmacol 2013;16:91-103. 
30. Roullet FI. Wollaston L, deCatanzaro D, Foster JA. Behavioral and molecular changes in the mouse in response to prenatal exposure to the anti-epileptic drug valproic acid. Neuroscience 2010;170: 514-22.

31. Monti B, Polazzi E, Contestabile A. Biochemical, molecular and epigenetic mechanisms of valproic acid neuroprotection. Curr Mol Pharmacol 2009;2:95-109.

32. Kim SH, Ko IG, Jin JJ, Hwang L, Baek SS. Treadmill exercise ameliorates impairment of spatial learning memory in pups born to old and obese mother rats. J Exerc Rehabil 2021;17:234-40. 